\title{
THREE-DIMENSIONAL EQUATIONS FOR MASS CENTRE MOTION OF AN AVALANCHE OF ARBITRARY CONFIGURATION
}

\author{
by \\ Yasuaki Nohguchi* \\ (Versuchsanstalt für Wasserbau, Hydrologie und Glaziologie, ETH, CH-8092 Zürich, Switzerland)
}

\section{ABSTRACT}

The path over a surface with complex topography that a snow avalanche will take is usually not known in advance. To predict avalanche paths over such terrain, we propose a model for the motion of the mass centre of an avalanche over an arbitrary surface. The numerical solution for this model shows that avalanche paths over complex topography are very sensitive to small differences in the avalanche velocity.

\section{INTRODUCTION}

Most avalanches which occur in valleys or mountainous regions with complex topography flow in unexpected directions. For example, the paths of avalanches in a valley with a curved thalweg do not always follow the thalweg itself (Fig. 1b); indeed, an avalanche may even pass over the ridge between two valleys and enter a neighbouring valley. Because of its inertia, an avalanche does not take the steepest descent path if these lines projected on to a horizontal plane are curved. In other words, the difference between the travel path and the steepest descent line depends on the velocity of the snow avalanche. In addition, over a convex surface (Fig. 1d) a small difference in the initial conditions sometimes yields significant differences in the travel path. Much theoretical work on snow-avalanche dynamics has been carried out, starting with that of Voellmy (1955), but there have been few studies of snow-avalanche paths, those existing being principally the work of Nohguchi (1983, 1986, 1987a, b). The problem of travel path is particularly notable for large-scale avalanches with long run-out distances and high speeds. If the length of the travel path is much longer than that of the main moving mass, we may represent the avalanche motion as that of a point mass or mass centre. In this paper, we formulate a three-dimensional model for the motion of the avalanche and use it to examine the effects of topography and velocity on the avalanche travel paths.

\section{GOVERNING EQUATIONS FOR MASS CENTRE MOTION}

\section{General description}

The first problem is to derive the equations for the motion of a point mass for an arbitrary surface. We do this using analytical mechanics (Goldstein, 1980). Locating the $x$ and $y$ axes in a horizontal plane (Fig. 2), an arbitrary surface is represented by the following equation

$$
z=f(x, y) .
$$

With this geometry, the equations of motion are

* Present address: Nagaoka Institute of Snow and Ice Studies, National Research Center for Disaster Prevention, Suyoshi, Nagaoka-shi 940, Japan.

$$
\begin{aligned}
& \frac{\mathrm{d} \dot{x}}{\mathrm{~d} t}=-\frac{f_{x}}{\left[1+f_{x}^{2}+f_{y}^{2}\right]^{\prime}} g^{\prime}-\frac{R}{m} \frac{\dot{x}}{V} \\
& \frac{\mathrm{d} \dot{y}}{\mathrm{~d} t}=-\frac{f_{y}}{\left[1+f_{x}^{2}+f_{y}^{2}\right]} g^{\prime}-\frac{R}{m} \frac{\dot{y}}{V}
\end{aligned}
$$

where $V$ and $R$ are the magnitude of the velocity and resistance repectively, and

$$
\begin{gathered}
g^{\prime}=f_{x x} \dot{x}^{2}+2 f_{x y} \dot{x} \dot{y}+f_{y y} \dot{y}^{2}+g, \\
f_{x}=\partial f / \partial x ; \quad f_{y}=\partial f / \partial y ; \\
f_{x x}=\partial^{2} f / \partial x^{2} ; \quad f_{y y}=\partial^{2} f / \partial y^{2} ; \quad f_{x y}=\partial^{2} f / \partial x \partial y .
\end{gathered}
$$

Equations (2a,b) describe the horizontal components of the mass centre motion. The $z$ component of the motion can be obtained from Equation (1). Equations (2a, b) can be solved by specifying the geometry in Equation (1), resistance force, $R$, and the initial conditions $(x, y, \dot{x}, \dot{y})=$ $\left(x_{0}, y_{0}, \dot{x}_{0}, \dot{y}_{0}\right)$.

Force of restriction and jump condition

The force due to the restriction condition in Equation (1) is equivalent to the normal force on the avalanche mass from the ground surface. Let $N_{x}, N_{y}$, and $N_{z}$ be the $x, y$, and $z$ components of the restriction force. Then we have

$$
\begin{aligned}
m \mathrm{~d} \dot{x} / \mathrm{d} t & =N_{x}-R \dot{x} / V \\
m \mathrm{~d} \dot{y} / \mathrm{d} t & =N_{y}-R \dot{y} / V \\
m \mathrm{~d} \dot{z} / \mathrm{d} t & =N_{z}-R \dot{z} / V-m g .
\end{aligned}
$$

By comparing Equations $(5 a, b, c)$ with Equations $(2 a, b)$ we have

$$
\begin{aligned}
& N_{x}=-m f_{x}\left(1+f_{x}^{2}+f_{y}^{2}\right)^{-1} g^{\prime} \\
& N_{y}=-m f_{y}\left(1+f_{x}^{2}+f_{y}^{2}\right)^{-1} g^{\prime} \\
& N_{z}=m\left(1+f_{x}^{2}+f_{y}^{2}\right)^{-1} g^{\prime} .
\end{aligned}
$$

Thus, the magnitude of the restriction force is

$$
N=m\left(1+f_{x}^{2}+f_{y}^{2}\right)^{-1 / 2}\left|g^{\prime}\right| .
$$

When either the velocity or ground-surface curvature is equal to zero, $g^{\prime}=g$, and $N=$ component of gravity normal to the surface.

In real configurations the ground is always below the avalanche surface and so $N_{z}$ must be positive to locate the point mass on the surface. The condition constraining the point mass to the surface is then 


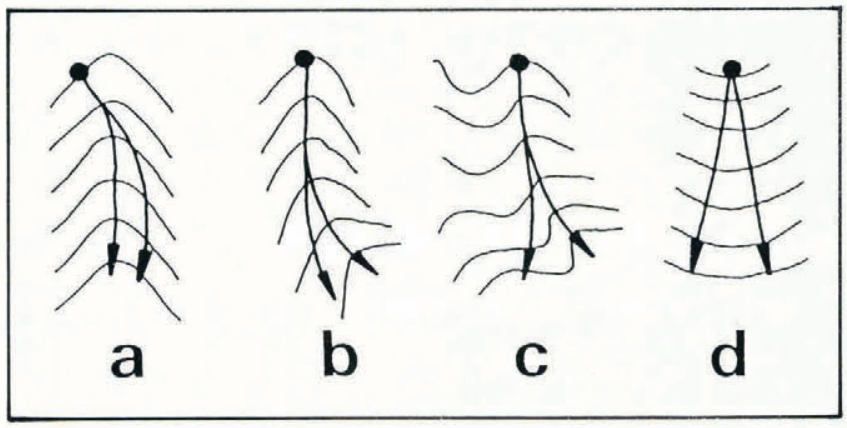

Fig. 1. Typical examples of avalanche travel paths. Thin lines are contours.

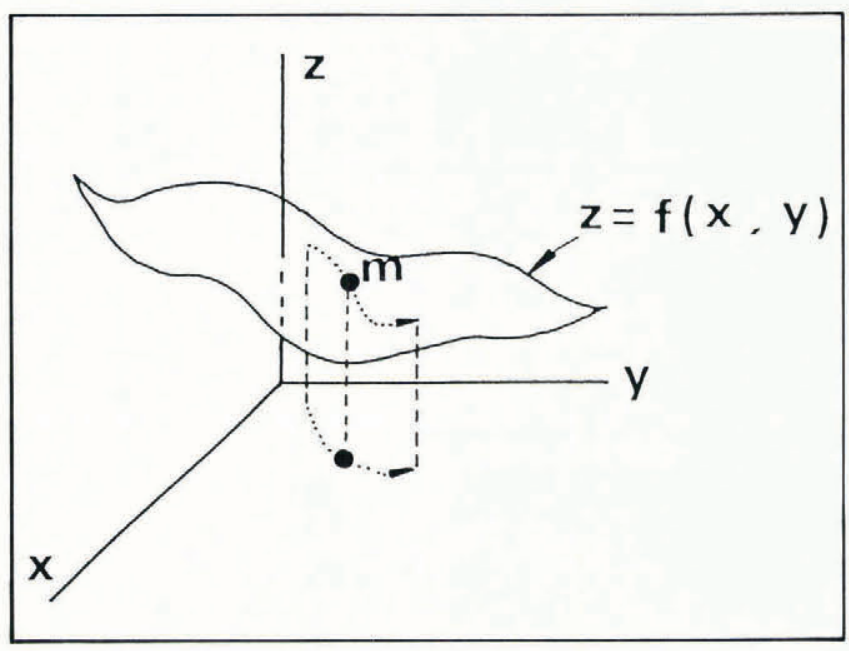

Fig. 2. Coordinate system and an arbitrary surface.

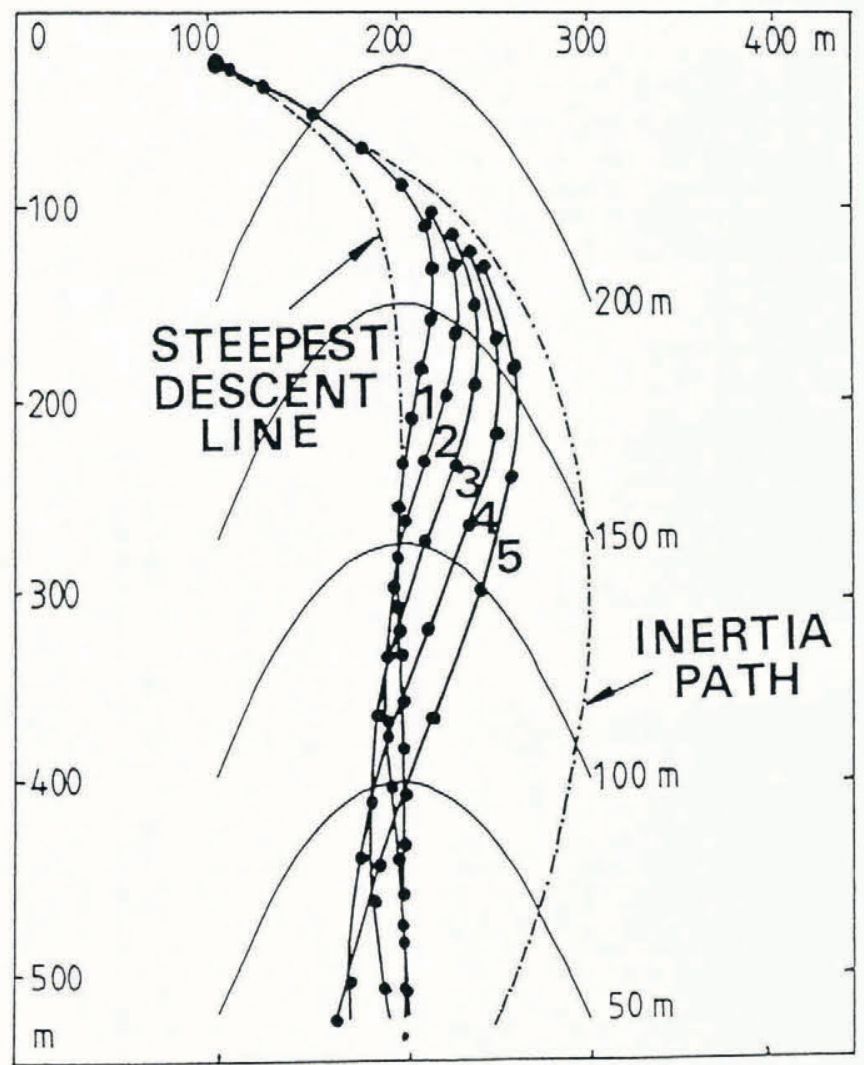

Fig. 3. Travel paths on parabolic valley shapes. Solid circles show position of avalanche mass centre at $2 \mathrm{~s}$ intervals. $\left(\mu=0.2 ;\right.$ (1) $\delta=0.01 \mathrm{~m}^{-1}, \quad V_{30}=17.9 \mathrm{~m} / \mathrm{s} ; \quad$ (2) $\delta=$ $0.005 \mathrm{~m}^{-1}, \quad V_{30}=25.3 \mathrm{~m} / \mathrm{s} ; \quad(3) \quad \delta=0.0025 \mathrm{~m}^{-1}, \quad V_{30}=$ $35.8 \mathrm{~m} / \mathrm{s}$; (4) $\delta=0.001 \mathrm{~m}^{-1}, V_{30}=56.6 \mathrm{~m} / \mathrm{s}$; (5) $\delta=0.0$; $V_{30}$ is $V_{\mathrm{e}}$ on a $30^{\circ}$ inclined plane slope.)

$$
g^{\prime}=g+f_{x x} \dot{x}^{2}+2 f_{x y} \dot{x} \dot{y}+f_{y y} \dot{y}^{2} \geqslant 0
$$

and we solve Equations (2a, b) subject to this constraint.

\section{Two particular travel paths}

We consider two distinct types of travel path independent of resistance forces. The first case assumes that inertia is important, so that

$$
\begin{aligned}
& \mathrm{d} \dot{x} / \mathrm{d} t=-f_{x}\left(1+f_{x}^{2}+f_{y}^{2}\right)^{-1} g^{\prime} \\
& \mathrm{d} \dot{y} / \mathrm{d} t=-f_{y}\left(1+f_{x}^{2}+f_{y}^{2}\right)^{-1} g^{\prime} .
\end{aligned}
$$

We call this the inertia path.

The other case assumes that inertia is not important and that therefore $\mathrm{d} \dot{x} / \mathrm{d} t=\mathrm{d} \dot{y} / \mathrm{d} t=0$, which gives

$$
\mathrm{d} y / \mathrm{d} x=f_{y} / f_{x}
$$

This path is the line of steepest descent, and for this reason we call it the steepest descent path. When the steepest descent path is straight, it is equivalent to the inertia path, and so every travel path is straight; the complication arises when the steepest descent path is curved.

\section{RESISTANCE FORCE}

In order to solve Equations $(2 \mathrm{a}, \mathrm{b})$ it is necessary to give concrete form to the resistance force. The form of this force is the major problem related to snow-avalanche modelling (Voellmy, 1955; Salm, 1966; Schaerer, 1975; Perla and others, 1980; Lang and others, 1985; Salm and Gubler, 1985; Maeno and Nishimura, 1987; Norem and others, 1987). For simplicity, in this paper we use the classical twoparameter representation

$$
R=\mu N+m \delta V^{2}
$$

where $\mu N$ is the frictional force; $\mu$ is the coefficient of friction; $m \delta V^{2}$ is the turbulence term; $\delta$ is the constant related to Voellmy's parameter $\xi(\delta=g / \xi h$, where $h$ is the flow height) or $=D / M$ in Perla's model. With $R$ given by Equation (11), Equations (2a, b) include only two parameters, $\mu$ and $\delta$.

A one-dimensional equation of motion on a plane slope is obtained from Equations $(2 a, b)$ and (11) as

$$
\mathrm{d} V / \mathrm{d} t=g(\sin \theta-\mu \cos \theta)-\delta V^{2}
$$

where $\theta$ is the slope angle. Following this, the terminal velocity on the plane slope can be given by

$$
V_{\mathrm{e}}=(g(\sin \theta-\mu \cos \theta) / \delta)^{\frac{1}{2}}
$$

in terms of $\mu$ and $\delta$, and $V_{\mathrm{e}}$ considered as a characteristic velocity scale.

\section{NUMERICAL COMPUTATIONS}

Figure 3 shows the results of numerical computations on a parabolic valley shape described by the following equation

$$
f(x, y)=\frac{a}{2} x^{2}+b y
$$

where $a$ represents the "steepness" of the valley, and $b$ the inclination of the thalweg. Using Equation (14), $f_{x}, f_{y}$, $f_{x x}, f_{x y}$, and $f_{y y}$ are given by

$f_{x}=a x ; \quad f_{y}=b ; \quad f_{x x}=a ; \quad f_{x y}=0 ; \quad f_{y y}=0$. 
With this geometry, we use a Runge-Kutta technique to solve Equations $(2 \mathrm{a}, \mathrm{b})$. The results show that the travel path depends strongly on $V_{\mathrm{e}}$; in particular, the deviation of the travel path from the steepest descent line increases with $V_{\mathrm{e}}$. Other results have been given by Nohguchi (1983).

Figure 4 shows the results of numerical computations using the topography of a slope in Japan where a largescale avalanche occurred on 26 January 1986. The path of this avalanche was about $2 \mathrm{~km}$ in length and curved smoothly from the release zone to the run-out zone. In

these calculations, $f_{x}, f_{y}, f_{x x}, f_{x y}$, and $f_{y y}$ were obtained from a contour map of the slope as follows

$$
\begin{aligned}
f_{x} & =\frac{f(x+\Delta, y)-f(x-\Delta, y)}{2 \Delta} \\
f_{y} & =\frac{f(x, y+\Delta)-f(x, y-\Delta)}{2 \Delta} \\
f_{x x} & =\frac{f(x+\Delta, y)-2 f(x, y)+f(x-\Delta, y)}{\Delta^{2}}
\end{aligned}
$$

$$
f_{x y}=\frac{f(x+\Delta, y+\Delta)-f(x-\Delta, y+\Delta)-f(x+\Delta, y-\Delta)+f(x-\Delta, y-\Delta)}{4 \Delta^{2}}
$$

$$
f_{x y}=\frac{f(x, y+\Delta)-2 f(x, y)+f(x, y-\Delta)}{\Delta^{2}}
$$

where, after considering the scale of the avalanche moving mass, $50 \mathrm{~m}$ was used as a value for $\Delta$. The computational results show that the avalanche path is dependent on the parameters listed in Table I; in particular, the avalanche path in run 2 is quite different from that in run 3 , even though $V_{\mathrm{e}}$ is only slightly different. Note that runs 3 and 4 are curved along the real avalanche path and extend over the cross-hatched region where the disaster occurred. Therefore, the real motion of the avalanche was accurately simulated by the motions of these runs.

Field data of run-out distance are frequently used to estimate model parameters (Martinelli and others, 1980). We have shown that it is also possible to calculated these parameters and to simulate the avalanche mass-centre motion by using the predicted travel path.

\section{TABLE I. MODEL PARAMETERS FOR EACH} RUN OVER REAL SURFACES

Run No.

$\mu$

0
0
0
0
0

0.002

0.0025

0.007

0.05

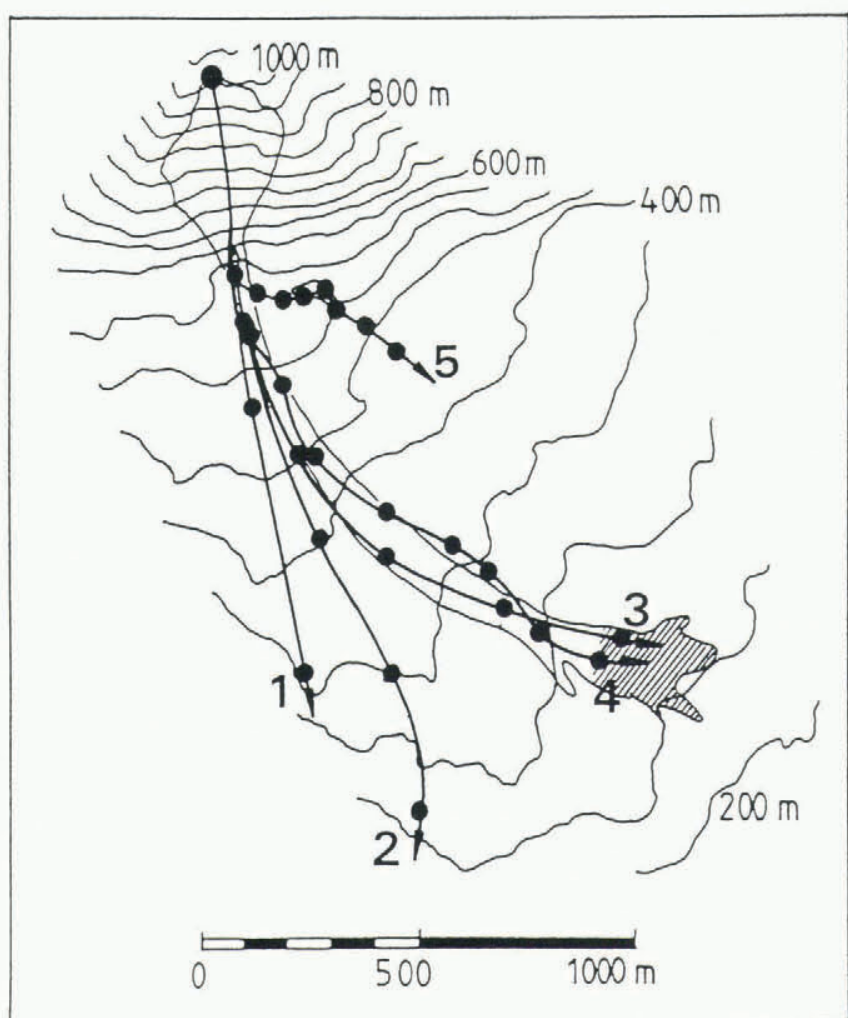

Fig. 4. Travel paths over real surfaces. Solid circles show position of avalanche mass centre at $10 \mathrm{~s}$ intervals.

real configuration. I. [In Japanese.] Rep. Natl. Res. Cent. Disaster Prevention, 38, 147-168.

Nohguchi, Y. 1987a. Equation for avalanche motion restricted by a diversion barrier. [In Japanese.] Rep. Natl. Res. Cent. Disaster Prevention, 39, 153-162.

Nohguchi, Y. 1987b. Traveling path of snow avalanche on real configuration. II. [In Japanese.] Rep. Natl. Res. Cent. Disaster Prevention, 39, 133-152.

Norem, H., F. Irgens, and B. Schieldrop. 1987. A continuum model for calculating snow avalanche velocities. International Association of Hydrological Sciences 162 (Symposium at Davos 1986 - Avalanche Formation, Movement and Effects), 363-379.

Perla, R., T.T. Cheng, and D.M. McClung. 1980. A twoparameter model of snow-avalanche motion. J. Glaciol., 26(94), 197-207.

Salm, B. 1966. Contribution to avalanche dynamics. International Association of Scientific Hydrology Publication 69 (Symposium at Davos 1965 - Avalanches and Physics of Snow), 199-214.

Salm, B. and H. Gubler. 1985. Measurement and analysis of the motion of dense flow avalanches. Ann. Glaciol., 6, 26-34.

Schaerer, P.A. 1975. Friction coefficients and speed of flowing avalanches. International Association of Hydrological Sciences Publication 114 (Symposium at Grindelwald 1974 - Snow Mechanics), 425-432.

Voellmy, A. 1955. Über die Zerstörungskraft von Lawinen. Schweiz. Bauztg., 73(12), 159-162; (15), 212-217; (17), 246-249; (19), 280-285. 